\title{
AN INVERSION THEOREM FOR THE STIELTJES TRANSFORM OF DISTRIBUTIONS
}

\author{
by STEVAN PILIPOVIĆ
}

(Received 28th August 1984)

\section{Introduction}

The Stieltjes transformation of generalized functions was investigated by Benedetto [1], Zemanian [16], Misra [10], Pandey [11], Lavoine and Misra [7], and Erdélyi [5]. The inversion theorems for the Stieltjes transform of generalized functions, in their own approaches, were given in Benedetto [1], Zemanian [16], Pandey [11], Pathak [12] (he used Pandey's approach) and Erdélyi [5].

We notice that the inversion theorems for the Stieltjes transform of functions were given in Widder [15], Sumner [13], Hirschman and Widder [6] and Byrne and Love [2], [9].

In this paper we use the definition of the distributional Stieltjes transform of index $\rho$ $\left(\rho \in \mathbb{R} \backslash\left(-\mathbb{N}_{0}\right) ; \mathbb{N}_{0}=\mathbb{N} \cup\{0\}\right), \quad S_{\rho}$-transform, given by Lavoine and Misra. The $S_{\rho^{-}}$ transform is defined for a subspace of Schwartz space $\mathscr{D}^{\prime}(\mathbb{R})$ while in [16], [10], [11] and [5] the Stieltjes transform is defined for the elements of appropriate spaces of generalized functions. But in these spaces the differentiation is not defined. This implies that, for example, the Stieltjes transform of $\delta^{(k)}(x-a), a \geqq 0, k \in \mathbb{N}$, is meaningless in the sense of [16], [10], [11], [5]. (The space investigated in [1] does not contain $\delta^{(k)}(x-a)$, $a \geqq 0$, as well.) It is easy to see that the $S_{\rho}$-transform of $\delta^{(k)}(x-a), a \geqq 0$, exists for $\rho>-k, \rho \in \mathbb{R} \backslash\left(-\mathbb{N}_{0}\right)$. This is the reason why we use the definition of the Stieltjes transform given in [7] and [8]. Carmichael and Milton [3] also used this definition for their investigations of the Stieltjes transform.

We are going to give the Inversion Theorem for the $\rho$-th Stieltjes transform of $f \in \mathscr{S}^{\prime}+$ (which has $\rho$-th Stieltjes transform) where $\mathscr{S}^{\prime}+$ is the space of tempered distributions with supports in $[0, \infty)$. We denote the $S_{\rho}$-transform of $f$ by $S_{\rho} f . S_{\rho} f$ is a holomorphic function in $\mathbb{C} \backslash(-\infty, 0]$ so we can apply, roughly speaking, the classical inversion formulae in order to obtain the properties of $f$ in the interval $(0, \infty)$. In the general case the point zero brings some difficulties for the full characterization of $f$. We overcome these difficulties by giving the distributional sense to the classical inversion formulae.

\section{Definition of the $S_{p}$-transform and notation}

Lavoine and Misra defined the Stieltjes transform by introducing spaces $\mathscr{D}^{\prime}(a)$ in [7] and $\mathscr{J}^{\prime}(r)$ in [8]. We give here the definition of the $S_{p}$-transform in a little different notation. 
The space $\mathscr{J}^{\prime}(\rho), \rho \in \mathbb{R} \backslash\left(-\mathbb{N}_{0}\right)$ is the space of distributions with supports in $[0, \infty)$ such that $f \in \mathscr{J}^{\prime}(\rho)$ iff there exist $k \in \mathbb{N}_{0}$ and a locally integrable function $F$ with $\operatorname{supp} F \subset[0, \infty)$ such that

(a) $f=D^{k} F$

(b) $\int_{0}^{\infty}|F(t)|(t+\beta)^{-\rho-k} d t<\infty$ for $\beta>0$

( $D$ is the distributional derivative).

If instead of (b) we suppose that there exist $C=C(F)$ and $\varepsilon=\varepsilon(F)>0$ such that

(c) $|F(x)| \leqq C(1+x)^{\rho+k-1-\varepsilon} \quad$ if $\quad x \geqq 0$,

the corresponding space is denoted by $\mathscr{I}^{\prime}(\rho)$. The space $\mathscr{I}^{\prime}(\rho)$ has the main role in this article.

Obviously, $\mathscr{I}^{\prime}(\rho) \subset \mathscr{J}^{\prime}(\rho), \rho \in \mathbb{R}\left(-\mathbb{N}_{0}\right) \mathbb{R} \backslash(-\mathbb{N})$. Let $f \in \mathscr{J}^{\prime}(\rho)$ and (1) (b) hold with $\rho+k>0$. If

$$
F_{1}(x)=\int_{0}^{x} F(t) d t, \quad x \in \mathbb{R}
$$

then

$$
\begin{aligned}
\left|F_{1}(x)\right|(1+x)^{-\rho-k} & \leqq(1+x)^{-\rho-k} \int_{0}^{x}|F(t)| d t \\
& \leqq \int_{0}^{x}|F(t)|(1+t)^{-\rho-k} d t \leqq M, \quad x \geqq 0,
\end{aligned}
$$

where

$$
M=\int_{0}^{\infty}|F(t)|(1+t)^{-\rho-k} d t<\infty
$$

Thus we have

$$
f=D^{k+1} F_{1}, \operatorname{supp} F_{1} \subset[0, \infty)
$$

and

$$
\left|F_{1}(x)\right| \leqq M(1+x)^{\rho+k}, \quad x \geqq 0
$$

If $\tilde{\rho}>\rho$ and $0<\varepsilon<\tilde{\rho}-\rho$ then

$$
\left|F_{1}(x)\right| \leqq M(1+x)^{\rho+(x+1)-1-\varepsilon}, \quad x \geqq 0 .
$$


This means that the following implication holds:

$$
f \in \mathscr{J}^{\prime}(\rho), \rho+k>0 \Rightarrow f \in \mathscr{I}^{\prime}(\tilde{\rho}) \text { for } \quad \tilde{\rho}>\rho, \quad \tilde{\rho} \in \mathbb{R} \backslash\left(-\mathbb{N}_{0}\right) \text {. }
$$

If $f \in \mathscr{J}^{\prime}(\rho)$ and (1)(b) holds with $\rho+k<0$, the situation is a little more complicated. Since

$$
\int_{0}^{\infty}|F(t)|(t+\beta)^{-p-k} d t<\infty
$$

it follows that

$$
\int_{x}^{\infty}|F(t)| d t<\infty, \quad x \geqq 0
$$

We put

$$
G(x):= \begin{cases}0, & x \leqq 0 \\ -\int_{x}^{\infty} F(t) d t+\alpha_{0} H(x), & x>0\end{cases}
$$

where $H(x)$ is the Heaviside function and $\alpha_{0}=\int_{0}^{\infty} F(t) d t$. In the distributional sense we have

$$
D G(x)=F(x) \text { and } f=D^{k+1} G
$$

If

$$
F_{1}(x):=-\int_{x}^{\infty} F(t) d t, \quad x \geqq 0
$$

then for $x \geqq 0$

$$
\begin{aligned}
\left|F_{1}(x)(1+x)^{-\rho-k}\right| & \leqq \int_{x}^{\infty}|F(t)|(1+t)^{-\rho-k} d t \\
& \leqq \int_{0}^{\infty}|F(t)|(1+t)^{-\rho-k} d t=M<\infty
\end{aligned}
$$

Thus, if $x \geqq 0$

$$
\begin{gathered}
|G(x)| \leqq M+\left|\alpha_{0}\right|, \text { i.e. } \\
|G(x)| \leqq\left(M+\left|\alpha_{0}\right|\right)(1+x)^{-(k+1)+(k+1)}
\end{gathered}
$$

and the following implication holds:

$$
f \in \mathscr{J}^{\prime}(\rho), \rho+k<0 \Rightarrow f \in \mathscr{I}^{\prime}(\tilde{\rho}) \quad \text { for } \quad \tilde{\rho}>-k \text { and } \tilde{\rho} \in \mathbb{R} \backslash\left(-\mathbb{N}_{0}\right)
$$


Now we define the Stieltjes transform $S_{\rho}, \rho \in \mathbb{R} \backslash\left(-\mathbb{N}_{0}\right)$, of a distribution $f \in \mathscr{J}^{\prime}(\rho)$ with properties given in (1). It is a complex-valued function given by

$$
\left(S_{\rho} f\right)(s):=(\rho)_{k} \int_{0}^{\infty} \frac{F(t) d t}{(t+s)^{\rho+k}}, \quad s \in \mathbb{C} \backslash(-\infty, 0]
$$

where $(\rho)_{k}=\rho(\rho+1) \ldots(\rho+k-1), k>0$ and $(\rho)_{0}=1$.

It is proved $([8])$ that $S_{\rho} f$ is a holomorphic function in $\mathbb{C} \backslash(-\infty, 0], \mathscr{J}^{\prime}(\rho) \subset \mathscr{J}^{\prime}(\rho+p)$, $p \in \mathbb{N}_{0}$, and

$$
(\rho)_{p}\left(S_{\rho+p} f\right)(s)=\left(S_{\rho}\left(f^{(p)}\right)\right)(s), \quad f \in \mathscr{J}^{\prime}(\rho+p)
$$

( $f^{(p)}$ also denotes $p$-th derivative).

Thus, if $f \in \mathscr{J}^{\prime}(\rho+p)$ then $f^{(p)} \in \mathscr{J}^{\prime}(\rho)$.

If $f$ is a tempered distribution with supp $f \subset[0, \infty)$ it is well known ([14] Ch. I, 5.4) that there exist $k \in \mathbb{N}_{0}, m \in \mathbb{N}_{0}$ and a unique $f_{k} \in L_{\text {loc }}^{1}(\mathbb{R})$ with $\operatorname{supp} f_{k} \subset[0, \infty)$ such that

$$
f=\left(f_{k}\right)^{(k)} \text { and } \int_{0}^{\infty}\left|f_{k}(t)\right|(1+t)^{-m} d t<\infty
$$

Thus, for any $f \in \mathscr{S}_{+}^{\prime}$ there are $k \in \mathbb{N}_{0}$ and $F \in L_{\text {loc }}^{1}(\mathbb{R})$ such that (1)(a) holds and there exists $\rho \in \mathbb{R} \backslash\left(-\mathbb{N}_{0}\right)$ such that $(1)\left(\right.$ b) holds as well. This means that any $f \in \mathscr{S}_{+}^{\prime}$ has the Stieltjes transform of index $\rho, \rho \in \mathbb{R} \backslash\left(-\mathbb{N}_{0}\right)$ for $\rho>\rho_{f}$ where

$$
\rho_{f}=\inf \left\{\rho \in \mathbb{R} \backslash\left(-\mathbb{N}_{0}\right) \text { such that (1) holds }\right\} \text {. }
$$

One can easily find distributions $f, g \in \mathscr{S}^{\prime}{ }_{+}$such that:

$$
\begin{aligned}
& \rho_{f} \in \mathbb{R} \backslash\left(-\mathbb{N}_{0}\right) \text { and } S_{\rho} f \text { does not exist for } \rho=\rho_{f} ; \\
& \rho_{g} \in \mathbb{R} \backslash\left(-\mathbb{N}_{0}\right) \text { and } S_{\rho} g \text { exists for } \rho=\rho_{g} .
\end{aligned}
$$

$\left(f(x)=H(x), g(x)=H(x-2) \ln ^{-2} x\right.$.)

We shall use the following differential operators from [5] (see [5], relation (6.1) with $p=1+n, q=1+n$ and $\rho+k$ instead of $\rho$ ):

$$
\mathscr{L}_{n, \rho, k, x}:=\frac{(-1)^{n+1} \Gamma(\rho+k)}{(n+1) ! \Gamma(n+\rho+k)} D^{n+1} x^{2 n+\rho+k+1} D^{n+1}, \quad n \in \mathbb{N}_{0}
$$

where $D$ is the ordinary derivative. We notice that these operators and similar ones are used in [9] as well. The operators $\mathscr{L}_{n, \rho, k, x}, n \in \mathbb{N}_{0}$, are formally self-adjoint.

Also, we shall use somewhat modified operators:

$$
L_{n, \rho, k, x}:=\frac{(-1)^{n+1} \Gamma(\rho+k+1)}{(n+1) ! \Gamma(n+\rho+k+1)(\rho)_{k+1}} D^{n+k+2} x^{2 n+\rho+k+2} D^{n+1}, \quad n \in \mathbb{N}_{0}
$$


As we are going to use these operators in the distributional sense with $D$ distributional derivative, we use the notation $\overline{\mathscr{L}}_{n, \rho, k, x}, \bar{L}_{n, \rho, k, x}$ if $D$ is understood in the distributional sense and $\mathscr{L}_{n, \rho, k, x}, \mathrm{~L}_{n, \rho, k, x}$ if $D$ is understood in the ordinary sense. Clearly, if a function $f$ has got "good" properties on some open interval then there is no difference between $\overline{\mathscr{L}}_{n, \rho, k, x}, \bar{L}_{n, \rho, k, x}$ and $\mathscr{L}_{n, \rho, k, x}, L_{n, \rho, k, x}$.

We shall use the following two formulae from [5] ((6.3) and (6.4)):

$$
\mathscr{L}_{n, \rho, k, x}(x+t)^{-\rho-k}=\frac{\Gamma(2 n+\rho+k+2)}{(n+1) ! \Gamma(n+\rho+k)} \frac{x^{n+\rho+k} t^{n+1}}{(x+t)^{2 n+\rho+k+2}}, \quad x \geqq 0, \quad t>0,
$$

and

$$
\int_{0}^{\infty} \mathscr{L}_{n, \rho, k, x}(x+t)^{-\rho-k} d t=1, \quad x>0, \quad n+\rho+k>0 .
$$

Except for two remarks at the end of the paper, we suppose that $f \in \mathscr{I}^{\prime}(\rho)$ i.e. $f=D^{k} F$ for some $F \in L_{\text {loc }}^{1}(\mathbb{R})$ with supp $f \subset[0, \infty)$ and some $k \in \mathbb{N}_{0}$ such that (c) holds.

\section{Inversion theorem}

We shall prove:

Inversion Theorem. Let $f \in \mathscr{I}^{\prime}(\rho)$. Then for any $\phi \in \mathscr{S}$

$$
\lim _{n \rightarrow \infty}\left\langle\bar{L}_{n, \rho, k, x}\left(\overline{S_{\rho} f}\right)_{+}(x), \phi(x)\right\rangle=\langle f(x), \phi(x)\rangle
$$

where $k$ is the value from (c).

By $\left(\overline{S_{\rho} f}\right)_{+}$we denote the distribution which correspond to the function $\left(S_{\rho} f\right)_{+}$where

$$
\left(S_{\rho} f\right)_{+}(x):= \begin{cases}\left(S_{\rho} f\right)(x) & x>0 \\ 0 & x \leqq 0\end{cases}
$$

If $\left(S_{\rho} f\right)_{+}$is a function from $L_{100}^{1}(\mathbb{R})$, then $\left(\overline{S_{\rho} f}\right)_{+}$denotes the regular distribution which corresponds to $\left(S_{\rho} f\right)_{+}$and if $\left(S_{\rho} f\right)_{+}$does not belong to $L_{100}^{1}(\mathbb{R})$, then $\left(\overline{S_{\rho} f}\right)_{+}$is an appropriate regularization of $\left(S_{\rho} f\right)_{+}$. This will be explained in detail in the following two sections.

If we compare our Inversion Theorem with the corresponding ones in Pandey [11] and Pathak [12], for example, we notice the following: the set $S$ of restrictions of functions in $\mathscr{S}=\mathscr{S}(\mathbb{R})$ on $(0, \infty)$ with the topology induced by that of $\mathscr{S}$ is a proper subspace of the testing function space $S_{\alpha}(0, \infty)$ in their notation. Hence the restrictions of elements from $S_{\alpha}^{\prime}(0, \infty)$ belong to $S^{\prime}$. Further, our Inversion Theorem is valid for $\phi \in \mathscr{S}$ whereas Pandey and Pathak use $\phi \in \mathscr{D}(0, \infty)$.

In proving the theorem we observe separately cases $\rho+k>0$ and $\rho+k<0$. 


\section{The case $\rho+k>0$}

Let us put $F_{1}(x)=\int_{0}^{x} F(t) d t, x \in \mathbb{R}$. The function $F_{1}$ is continuous, $F_{1}(0)=0$ and $F_{1}^{(1)}=F$. It follows from (c) that for some $C_{1}>0$

$$
\left|F_{1}(x)\right| \leqq \int_{0}^{x}|F(t)| d t \leqq C_{1}(1+x)^{\rho+k-\varepsilon}, \quad x \geqq 0
$$

(we choose $\varepsilon$ such that $\rho+k-\varepsilon>0$ ).

Since $f=F_{1}^{(k+1)}$ from (6), by partial integration, we obtain

$$
\left(S_{\rho} f\right)(s)=(\rho)_{k+1}\left(S_{\rho+k+1} F_{1}\right)(s), \quad s \in \mathbb{C} \backslash(-\infty, 0]
$$

In the same way as in [5], p. 239 (proof of $L_{n, x} \hat{\phi} \rightarrow \phi(x)$ as $n \rightarrow \infty$ ) we can prove that for $x>0$

$$
\mathscr{L}_{n, \rho, k+1, x}\left(S_{\rho+k+1} F_{1}\right)(x) \rightarrow F_{1}(x) \text { as } n \rightarrow \infty
$$

We want to obtain an estimate of the convergence rate in (7). Because of that, in the following lemma, we give the complete proof of (7).

Lemma 1. The sequence

$$
\frac{\mathscr{L}_{n, \rho, k+1, x}\left(S_{\rho+k+1} F_{1}\right)(x)-F_{1}(x)}{x B(x)}, \quad n \in N,
$$

where $B(x)=(1+x)^{\rho+k-1-\varepsilon}$ if $\rho+k-1-\varepsilon>0$ and $B(x)=1$ if $\rho+k-1-\varepsilon<0, x \in(0, \infty)$, converges uniformly to zero on $(0, \infty)$ as $n \rightarrow \infty$.

Proof. From (4), (5) and (c) it follows that:

$$
\begin{aligned}
& \left|\mathscr{L}_{n, \rho, k+1, x}\left(S_{\rho+k+1} F_{1}\right)(x)-F_{1}(x)\right| \\
& \quad=\frac{\Gamma(2 n+\rho+k+3)}{(n+1) ! \Gamma(n+\rho+k+1)}\left|\int_{0}^{\infty} \frac{\left(F_{1}(t)-F_{1}(x)\right) x^{n+\rho+k+1} t^{n+1}}{(x+t)^{2 n+\rho+k+3}} d t\right| \\
& \quad \leqq \frac{\Gamma(2 n+\rho+k+3)}{(n+1) ! \Gamma(n+\rho+k+1)}\left|\int_{0}^{\infty} \frac{\left(F_{1}(u x)-F_{1}(x)\right) u^{n+1}}{(1+u)^{2 n+\rho+k+3}} d u\right| \\
& \quad \leqq \frac{\Gamma(2 n+\rho+k+3) x}{(n+1) ! \Gamma(n+\rho+k+1)} \int_{0}^{\infty} \frac{u^{n+1}}{(1+u)^{2 n+\rho+k+3}}\left|\int_{1}^{u}\right| F_{1}^{\prime}(x s)|d s| d u \\
& \quad \leqq \frac{\Gamma(2 n+\rho+k+3) x}{(n+1) ! \Gamma(n+\rho+k+1)} \int_{0}^{\infty} \frac{u^{n+1}}{(1+u)^{2 n+\rho+k+3}}\left|\int_{1}^{u}\right| F(x s)|d s| d u
\end{aligned}
$$




$$
\begin{aligned}
& \leqq C \frac{\Gamma(2 n+\rho+k+3) x}{(n+1) ! \Gamma(n+\rho+k+1)} \int_{0}^{\infty} \frac{u^{n+1} A(x, u)|u-1|}{(1+u)^{2 n+\rho+k+3}} d u \\
& \leqq C \frac{\Gamma(2 n+\rho+k+3)}{(n+1) ! \Gamma(n+\rho+k+1)} x B(x) \int_{0}^{\infty} \frac{u^{n+1} A_{1}(u)|u-1|}{(1+u)^{2 n+\rho+k+3}} d u
\end{aligned}
$$

where for $\rho+k-1-\varepsilon>0$

$$
A(x, u):=\left\{\begin{array}{ll}
(1+x)^{\rho+k-1-\varepsilon}, & u \in(0,1) \\
(1+x u)^{\rho+k-1-\varepsilon}, & u \in[1, \infty)
\end{array}, \quad A_{1}(u):= \begin{cases}1, & u \in(0,1) \\
(1+u)^{\rho+k-1-\varepsilon}, & u \in[1, \infty)\end{cases}\right.
$$

and for $\rho+k-1-\varepsilon<0 A(x, u):=1, A_{1}(u):=1, x, u \in(0, \infty)$.

If we put

$$
\Lambda(u):=\frac{u A_{1}(u)|u-1|}{(1+u)^{\rho+k+3}}, \quad u \in(0, \infty)
$$

then $\Lambda$ satisfies the conditions of Widder's theorem ([15], p. 344, Theorem 8.c) which implies

$$
\frac{(2 n-1) !}{n !(n-2) !} \int_{0}^{\infty} \frac{u^{n} \Lambda(u)}{(1+u)^{2 n}} d u \rightarrow \Lambda(1)=0 \quad \text { as } \quad n \rightarrow \infty
$$

Since

$$
\frac{\Gamma(2 n+\rho+k+3) n !(n-2) !}{(2 n-1) !(n+1) ! \Gamma(n+\rho+k+1)} \rightarrow 2^{\rho+k+3} \text { as } n \rightarrow \infty
$$

the assertion of Lemma 1 follows.

Lemma 2. $x^{\rho+k}\left(S_{\rho} f\right)(x), x \in(0, \infty)$ is a bounded function in any interval $(0, B), B>0$.

Proof. For $x>0$ we have

$$
\begin{aligned}
& \left|x^{\rho+k} \int_{0}^{\infty} \frac{F_{1}(t)}{(x+t)^{\rho+k+1}} d t\right| \\
& \quad \leqq x^{\rho+k}\left(\int_{0}^{B} \frac{\left|F_{1}(t)\right|}{(x+t)^{\rho+k+1}} d t+\int_{B}^{\infty} \frac{\left|F_{1}(t)\right|}{(x+t)^{\rho+k+1}} d t\right) \\
& \quad \leqq x^{\rho+k} \int_{0}^{B} \frac{\left|F_{1}(t)\right|}{(x+t)^{\rho+k+1}} d t+x^{\rho+k} \int_{B}^{\infty} \frac{\left|F_{1}(t)\right|}{t^{\rho+k+1}} d t .
\end{aligned}
$$


Thus, we have to prove that

$$
x^{\rho+k} \int_{0}^{B} \frac{\left|F_{1}(t)\right|}{(x+t)^{\rho+k+1}} d t
$$

is bounded when $x$ tends to 0 . have

From (6) it follows that $\left|F_{1}(x)\right| \leqq C, x \in(0, B)$, where $C$ is a suitable constant. So we

$$
x^{\rho+k} \int_{0}^{B} \frac{\left|F_{1}(t)\right|}{(x+t)^{\rho+k+1}} d t \leqq C x^{\rho+k} \int_{0}^{B} \frac{1}{(x+t)^{\rho+k+1}} d t
$$

By the direct computation of the last integral the assertion follows.

Let us denote by $[\rho+k]$ the greatest integer not exceeding $\rho+k$. Since $[\rho+k]-(\rho+k)>-1$ and

$$
\left|x^{[\rho+k]}\left(S_{\rho} f\right)_{+}(x)\right|=\left|x^{[\rho+k]-[\rho+k)} x^{\rho+k}\left(S_{\rho} f\right)_{+}(x)\right| \leqq C x^{[\rho+k]-(\rho+k)},
$$

where $x \in(0, B), B>0$ and $C$ is a suitable constant, it follows that $x^{[\rho+k]}\left(S_{\rho} f\right)_{+}(x)$ is locally integrable on $\mathbb{R}$.

We put

$$
l:=[\rho+k]-1
$$

Obviously, the cases

$$
\left(S_{\rho} f\right)_{+} \in L_{\mathrm{loc}}^{1}(\mathbb{R}) \quad \text { or } \quad x^{s}\left(S_{\rho} f\right)_{+} \in L_{\mathrm{loc}}^{1}(\mathbb{R})
$$

are possible for some $s, 0<s<l+1$ which depends on $f$. Let us suppose the case (we call it the extreme case):

$$
x^{l}\left(S_{\rho} f\right)_{+}(x) \notin L_{\mathrm{loc}}^{1}(\mathbb{R})
$$

In this case $\left(\overline{S_{\rho} f}\right)_{+}$denotes the following regularization of the functions $\left(S_{\rho} f\right)_{+}$(see [4], p. 11):

$$
\begin{aligned}
& \left\langle\left(\overline{S_{\rho} f}\right)_{+}(x), \phi(x)\right\rangle:=\int_{0}^{1}\left(S_{\rho} f\right)(x)\{\phi(x)-\phi(0)-\cdots \\
& \left.\cdots-\frac{x^{l}}{l !} \phi^{(l)}(0)\right\} d x+\int_{1}^{\infty}\left(S_{\rho} f\right)(x) \phi(x) d x, \quad \phi \in \mathscr{S} .
\end{aligned}
$$

If we put

$$
\left(S_{\rho+k+1+i} F_{1}\right)_{+}(x):=\left\{\begin{array}{ll}
\left(S_{\rho+k+1+i} F_{1}\right)(x), & x>0 \\
0, & x \leqq 0
\end{array} \quad i \in \mathbb{N}_{0}\right.
$$


from the proof of Lemma 2 it follows that we have to put

$$
\begin{aligned}
& \left\langle\left(\overline{S_{\rho+k+1+i} F_{1}}\right)_{+}(x), \phi(x)\right\rangle: \\
& =\int_{0}^{1}\left(S_{\rho+k+1+i} F_{1}\right)(x)\left\{\phi(x)-\phi(0)-\cdots-\frac{x^{l+i}}{(l+i) !} \phi^{(l+i)}(0)\right\} d x \\
& \quad+\int_{1}^{\infty}\left(S_{\rho+k+1+i} F_{1}\right)(x) \phi(x) d x
\end{aligned}
$$

Let us find the relations between $\left(\overline{S_{\rho} f}\right)_{+}^{(m)}$ and $\left(\overline{S_{\rho+k+1+m} F_{1}}\right)+m \in \mathbb{N}$. For $m=1$ and $\phi \in \mathscr{I}$ we have

$$
\begin{aligned}
\left\langle\left(\overline{S_{\rho} f}\right)_{+}^{\prime}(x), \phi(x)\right\rangle= & -\left\langle\left(\overline{S_{\rho} f}\right)_{+}(x), \phi^{\prime}(x)\right\rangle \\
= & -\int_{0}^{1}\left(S_{\rho} f\right)(x)\left\{\phi^{\prime}(x)-\phi^{\prime}(0)-\cdots-\frac{x^{l} \phi^{(l+1)}(0)}{l !}\right\} d x \\
& -\int_{1}^{\infty}\left(S_{\rho} f\right)(x) \phi^{\prime}(x) d x \\
= & -(\rho)_{k+1} \int_{0}^{1}\left(S_{\rho+k+1} F_{1}\right)(x)\left\{\phi(x)-\phi(0)-\cdots-\frac{x^{l+1} \phi^{(l+1)}(0)}{(l+1) !}\right\} d x \\
& -(\rho)_{k+1} \int_{1}^{\infty}\left(S_{\rho+k+1} F_{1}\right)(x) \phi^{\prime}(x) d x \\
= & (\rho)_{k+1}\left(S_{\rho+k+1} F_{1}\right)(1)\left\{\phi(1)-\phi(0)-\cdots-\frac{\phi^{(l+1)}(0)}{(l+1) !}\right\} \\
& -(\rho)_{k+2} \int_{0}^{1}\left(S_{\rho+k+2} F_{1}\right)(x)\{\phi(x)-\phi(0)-\cdots \\
& \left.\cdots-\frac{x^{l+1} \phi^{(l+1)}(0)}{(l+1) !}\right\} d x \\
& +(\rho)_{k+1}\left(S_{\rho+k+1} F_{1}\right)(1) \phi(1)-(\rho)_{k+2} \int_{1}^{\infty}\left(S_{\rho+k+2} F_{1}\right)(x) \phi(x) d x
\end{aligned}
$$

Thus, we obtain

$$
\begin{aligned}
\left(\overline{S_{\rho} f}\right)_{+}^{\prime}= & -(\rho)_{k+2}\left(\overline{S_{\rho+k+2} F_{1}}\right)_{+}(x)+(\rho)_{k+1}\left(S_{\rho+k+1} F\right)(1) \\
& \sum_{i=0}^{l+1}(-1)^{i} \delta^{(i)} / i !
\end{aligned}
$$


and by repeating the preceding arguments we obtain

$$
\begin{gathered}
\left(\overline{S_{\rho} f}\right)_{+}^{(m)}=(-1)^{m}(\rho)_{k+1+m}\left(\overline{S_{\rho+k+1+m} F_{1}}\right)_{+}+A_{m, k}(\delta) \\
A_{m, k}(\delta)=\sum_{i=0}^{l+m} C_{m, k, i} \delta^{(i)}
\end{gathered}
$$

where $C_{m, k, i}, i=0, \ldots, l+m$, are suitable constants.

If $j \in \mathbb{N},(9)$ and (10) imply

$$
\begin{aligned}
\left(\overline{S_{\rho} f}\right)_{+}^{(m+j)} & =\left(\left(\overline{S_{\rho} f}\right)_{+}^{(m)}\right)^{(j)} \\
& =(-1)^{m}(\rho)_{k+1+m}\left(\overline{S_{\rho+k+1+m} F_{1}}\right)_{+}^{(j)}+\left(A_{m, k}(\delta)\right)^{(j)} \\
& =(-1)^{m+j}(\rho)_{k+1+m+j}\left(\overline{S_{\rho+k+1+m+j} F_{1}}\right)_{+}+A_{m+j, k}(\delta) .
\end{aligned}
$$

So we obtain

$$
\begin{gathered}
\overline{\left(S_{\rho+k+1+m} F_{1}\right)_{+}^{(j)}=}(-1)^{j}(\rho+k+1+m)_{j}\left(\overline{S_{\rho+k+1+m+j} F_{1}}\right)_{+} \\
+A_{m, k, j}(\delta), \\
A_{m, k, j}(\delta)=\sum_{i=0}^{l+m+j} C_{m, k, j, i} \delta^{(i)}
\end{gathered}
$$

where $C_{m, k, j, i}, i=0,1, \ldots, l+m+j$, are suitable constants.

If for some number $s, 0<[s]<l$, the following conditions hold:

$$
x^{[s]}\left(S_{\rho} f\right)_{+}(x) \notin L_{\mathrm{loc}}^{1}(\mathbb{R})
$$

and

$$
x^{[s]+1}\left(S_{\rho} f\right)_{+}(x) \in L_{\mathrm{loc}}^{1}(\mathbb{R})
$$

then in (8) resp. $\left(8^{\prime}\right)$ the last factor has to be

$$
\frac{x^{[s]}}{([s]) !} \phi^{([s])}(0) \quad \text { resp. } \quad \frac{x^{[s]+i}}{([s]+i) !} \phi^{([s]+i)}(0)
$$

In this case we obtain formulae similar to (9)-(11).

If $\left(S_{\rho} f\right)_{+} \in L_{\text {loc }}^{1}$, then $\left(\overline{S_{\rho} f}\right)_{+}$is a regular distribution. If in the computation of $\bar{L}_{n, \rho, k, x}\left(\overline{S_{\rho} f}\right)_{+}(n \rightarrow \infty)$ regularizations are to appear, then they have to be made in the way which we have already described. 
Proof of the Inversion Theorem for $\rho+k>0$. We shall give the proof for the extreme case i.e. we suppose

$$
x^{l}\left(S_{\rho} f\right)+\notin L_{10 c}^{1}(\mathbb{R})
$$

The other cases discussed above can be proved in a similar way. By (9) we obtain $(\phi \in \mathscr{S})$

$$
\begin{aligned}
\left\langle\bar{L}_{n, p, k, x}\left(\overline{S_{\rho} f}\right)_{+}(x), \phi(x)\right\rangle & \\
= & \frac{(-1)^{n+k} \Gamma(\rho+k+1)}{(n+1) ! \Gamma(n+\rho+k+1)(\rho)_{k+1}}\left\langle D^{n+1} x^{2 n+\rho+k+2} D^{n+1}\left(\overline{S_{\rho} f}\right)_{+}(x), \phi^{(k+1)}(x)\right\rangle \\
= & \frac{(-1)^{n+k} \Gamma(\rho+k+1)}{(n+1) ! \Gamma(n+\rho+k+1)(\rho)_{k+1}}\left\langleD ^ { n + 1 } x ^ { 2 n + \rho + k + 2 } \left((-1)^{n+1}(\rho)_{k+n+2}\right.\right. \\
& \left.\left.\cdot\left(\overline{S_{\rho+k+n+2} F_{1}}\right)_{+}(x)+A_{n+1, k}(\delta)(x)\right), \phi^{(k+1)}(x)\right\rangle .
\end{aligned}
$$

Using Leibniz formula, (11), the fact that $x^{p} \delta^{(k)}(x)=0$ if $p>k$ and $\left(8^{\prime}\right)$, we have

$$
\begin{aligned}
& \left\langle D^{n+1} x^{2 n+\rho+k+2} D^{n+1}\left(\overline{S_{\rho} f}\right)_{+}(x), \phi^{(k+1)}(x)\right\rangle \\
& =\left\langle\sum_{i=0}^{n+1}\left(\begin{array}{c}
n+1 \\
i
\end{array}\right)\left(x^{2 n+\rho+k+2}\right)^{(n+1-i)}\right. \\
& \cdot\left((-1)^{n+1}(\rho)_{k+n+2}\left(\overline{S_{\rho+k+n+2} F_{1}}\right)_{+}^{(i)}(x)\right. \\
& \left.\left.+\left(A_{n+1, k}(\delta)\right)^{(i)}(x)\right), \phi^{(k+1)}(x)\right\rangle \\
& =\left\langle\sum_{i=0}^{n+1}\left({ }^{n+1} i\right)\left(x^{2 n+\rho+k+2}\right)^{(n+1-i)}(-1)^{n+1+i}(\rho)_{k+n+2+i}\right. \\
& \left.\cdot\left(\overline{S_{p+k+n+2+i} F_{1}}\right)_{+}(x), \phi^{(k+1)}(x)\right\rangle \\
& =\sum_{i=0}^{n+1}(-1)^{n+1+i(n+1}\left(\begin{array}{c}
i \\
)
\end{array}\right)(2 n+\rho+k+2) \\
& \cdot \ldots \cdot(n+\rho+k+2+i)(\rho)_{k+n+i+2} \int_{0}^{\infty}\left(S_{\rho+k+n+i+2} F_{1}\right)(x) x^{n+\rho+k+i+1} \phi^{(k+1)}(x) d x
\end{aligned}
$$


Thus, we obtain

$$
\begin{aligned}
\left\langle\bar{L}_{n, \rho, k, x}\left(\overline{S_{\rho} f}\right)_{+}(x), \phi(x)\right\rangle \\
\quad=\sum_{i=0}^{n+1} \frac{(-1)^{i+k+1}\left({ }^{n+1}\right) \Gamma(\rho+k+1)(n+\rho+k+i+2)_{n+1-i}(\rho)_{k+n+i+2}}{(n+1) ! \Gamma(n+\rho+k+1)(\rho)_{k+1}} \\
\quad \cdot \int_{0}^{\infty}\left(S_{\rho+k+n+i+2} F_{1}\right)(x) x^{n+\rho+k+i+1} \phi^{(k+1)}(x) d x .
\end{aligned}
$$

For $x>0$ we have

$$
\begin{gathered}
\sum_{i=0}^{n+1}\left({ }^{n+1}{ }_{i}\right) \frac{(-1)^{i} \Gamma(\rho+k+1)(\rho+k+1)_{n+1+i}(n+\rho+k+i+2)_{n+1-i}}{(n+1) ! \Gamma(n+\rho+k+1)} \\
\cdot x^{n+\rho+k+i+1}\left(S_{\rho+k+n+i+2} F_{1}\right)(x)=\mathscr{L}_{n, \rho, k+1, x}\left(S_{\rho+k+1} F_{1}\right)(x) .
\end{gathered}
$$

Now Lemma 1 implies

$$
\begin{aligned}
& \left\langle\bar{L}_{n, \rho, k, k, x}\left(\overline{S_{\rho} f}\right)_{+}(x)-f(x), \phi(x)\right\rangle \\
& =(-1)^{k+1} \int_{0}^{\infty}\left(\mathscr{L}_{n, \rho, k+1, x}\left(S_{\rho+k+1} F_{1}\right)(x)-F_{1}(x)\right) \phi^{(k+1)}(x) d x \\
& =(-1)^{k+1} \int_{0}^{\infty} \frac{\mathscr{L}_{n, \rho, k+1, x}\left(S_{\rho+k+1} F_{1}\right)(x)-F_{1}(x)}{x B(x)} \\
& \quad \cdot\left(x B(x) \phi^{(k+1)}(x)\right) d x \rightarrow 0 \text { as } n \rightarrow \infty .
\end{aligned}
$$

\section{The case $\rho+k<0$}

Let us remark that $\rho+k \notin-\mathbb{N}_{0}$.

We put

$$
F_{1}(x):= \begin{cases}-\int_{x}^{\infty} F(t) d t, & x>0 \\ 0, & x \leqq 0\end{cases}
$$

Since we suppise that $f \in \mathscr{I}^{\prime}(\rho)$, (c) implies that there exists $B_{1}>0$ such that

$$
\left|F_{1}(x)\right| \leqq \int_{x}^{\infty}|F(t)| d t \leqq B_{1}(1+x)^{\rho+k+\varepsilon}, \quad x \geqq 0
$$

In the sense of the distributional derivative we have

$$
F_{1}^{\prime}=F-\alpha_{0} \delta, \quad \text { where } \quad \alpha_{0}=\int_{0}^{\infty} F(t) d t
$$


By partial integration we obtain $(x>0)$

$$
\begin{aligned}
\left(S_{\rho} f\right)(x) & =(\rho)_{k} \int_{0}^{\infty} \frac{F(t)}{(x+t)^{\rho+k}} d t \\
& =(\rho)_{k}\left((\rho+k) \int_{0}^{\infty} \frac{F_{1}(t)}{(x+t)^{\rho+k+1}} d t+\alpha_{0} \frac{1}{x^{\rho+k}}\right)
\end{aligned}
$$

Let

$$
x_{+}^{-\rho-k}:= \begin{cases}x^{-\rho-k}, & x>0 \\ 0, & x \leqq 0\end{cases}
$$

Lemma 3. If $\phi \in \mathscr{S}$ and $n \rightarrow \infty$ we have

$$
(\rho)_{k}\left\langle\vec{L}_{n, \rho, k, x^{-\rho-k}}, \phi(x)\right\rangle \rightarrow\left\langle\delta^{(k)}(x), \phi(x)\right\rangle
$$

Proof. Since $-\rho-k>0$ and $n \rightarrow \infty$, there exists $s \in \mathbb{N}_{0}$ such that in the expression $D^{n+1} x_{+}^{-p-k}$ ( $n$ sufficiently large) the regularizations of $x_{+}^{-\rho-k-j}, j=s, \ldots, n+1$ are to appear. We regularize these functions in the usual way ([4]). From the fact that $x^{p} \delta^{(j)}(x)=0$ if $p>j$, we obtain

$$
\begin{aligned}
\frac{(\rho)_{k}(-1)^{n+k} \Gamma(\rho+k+1)}{(n+1) ! \Gamma(n+\rho+k+1)(\rho)_{k+1}} \\
\cdot\left\langle D^{n+1} x^{2 n+\rho+k+2} D^{n+1} x_{+}^{-\rho-k}, \phi^{(k+1)}(x)\right\rangle \\
=\frac{(-1)^{n+k} \Gamma(\rho+k+1)}{(n+1) ! \Gamma(n+\rho+k+1)(\rho+k)} \\
\quad \cdot \int_{-\infty}^{\infty} D^{n+1}\left((-1)^{n+1}(\rho+k)_{n+1} x_{+}^{n+1}\right) \phi^{(k+1)}(x) d x \\
=-\frac{(-1)^{k} \Gamma(\rho+k+1)(\rho+k+1)_{n}}{\Gamma(n+\rho+k+1)}\left\langle H(x), \phi^{(k+1)}(x)\right\rangle \\
=\left\langle H^{(k+1)}(x), \phi(x)\right\rangle, \quad \phi \in \mathscr{S} .
\end{aligned}
$$

Similarly as in Lemma 1 (by using Widder's theorem [15], p. 344), one can prove:

\section{Lemma 4.}

$$
\frac{\mathscr{L}_{n, \rho, k+1, x}\left(S_{\rho+k+1} F_{1}\right)(x)-F_{1}(x)}{x}
$$


converges uniformly to zero on $(0, \infty)$ as $n \rightarrow \infty$.

Proof of the Inversion Theorem for $\rho+k<0$. Let $\phi \in \mathscr{S}$. If we prove

$$
\begin{aligned}
\lim _{n \rightarrow \infty}\left\langle\bar{L}_{n, \rho, k, x}(\rho)_{k+1}\left(\overline{S_{\rho+k+1} F_{1}}\right)_{+}(x), \phi(x)\right\rangle \\
\quad=\left\langle F_{1}^{(k+1)}(x), \phi(x)\right\rangle
\end{aligned}
$$

then (13), Lemma 3 and (12) imply

$$
\begin{aligned}
\lim _{n \rightarrow \infty}\left\langle\bar{L}_{n, \rho, k, x}\left(\overline{S_{\rho} f}\right)_{+}(x), \phi(x)\right\rangle \\
=\lim _{n \rightarrow \infty}\left\langle\bar{L}_{n, \rho, k, x}(\rho)_{k+1}\left(\overline{S_{\rho+k+1} F_{1}}\right)_{+}(x), \phi(x)\right\rangle \\
\quad+\alpha_{0} \lim _{n \rightarrow \infty}\left\langle\bar{L}_{n, \rho, k, x}(\rho)_{k} x_{+}^{-\rho-k}, \phi(x)\right\rangle \\
=\left\langle\left(F_{1}^{(1)}+\alpha_{0} \delta\right)^{(k)}, \phi\right\rangle=\left\langle F^{(k)}, \phi\right\rangle=\langle f, \phi\rangle .
\end{aligned}
$$

For the proof of (14) we have to repeat the arguments of the proof of the Inversion Theorem for $\rho+k>0$. The regularizations of $\left(S_{\rho+k+1} F_{1}\right)_{+}^{(j)}$ will occur for $0<s \leqq j \leqq n+1$ where $s$ does not depend on $n$. At the end, instead of Lemma 1 we have to use Lemma 4.

Thus, the Inversion Theorem is completely proved.

Now we give two remarks related to the space $\mathscr{J}^{\prime}(\rho)$.

Remark 1. Let us suppose that $f \in \mathscr{J}^{\prime}(\rho), \rho \in \mathbb{R} \backslash\left(-\mathbb{N}_{0}\right)$ such that (1) holds with $\rho+k>0$. According to Section 2 we have $f \in \mathscr{I}^{\prime}(\tilde{\rho})$ for $\tilde{\rho}>\rho, \tilde{\rho} \in \mathbb{R} \backslash\left(-\mathbb{N}_{0}\right)$. Let $\tilde{\rho}:=\rho+1$. From $\left(S_{\rho} f\right)^{\prime}=-\rho\left(S_{\rho+1} f\right)$ on $(0, \infty)$, we obtain

$$
-\frac{1}{\rho} \bar{L}_{n, \rho+1, k+1, x} D\left(\overline{S_{\rho} f}\right)_{+}(x) \rightarrow f(x) \text { in } \mathscr{S}^{\prime} \text { as } n \rightarrow \infty .
$$

Remark 2. Let $f \in \mathscr{J}^{\prime}(\rho)$ and (1) hold with $\rho+k<0$. Then, because of the fact that $f \in \mathscr{I}^{\prime}(\tilde{\rho}), \tilde{\rho}>-k, \tilde{\rho} \in \mathbb{R} \backslash\left(-\mathbb{N}_{0}\right)$, we choose the smallest $j \in \mathbb{N}_{0}$ such that $\rho+j>-k$. Now, from

$$
\left(S_{\rho} f\right)^{(j)}=(-1)^{j}(\rho)_{j}\left(S_{\rho+j} f\right) \text { on }(0, \infty)
$$

and

$$
f=\left(F_{1}+\alpha_{0} H\right)^{(k+1)} \text { on } \mathbb{R}(\text { see Section } 2),
$$


we obtain

$$
(-1)^{j} \frac{1}{(\rho)_{j}} L_{n, \rho+j, k+1, x} D^{j}\left(\overline{S_{\rho} f}\right)_{+}(x) \rightarrow f(x) \quad \text { in } \quad \mathscr{S}^{\prime} \text { as } n \rightarrow \infty
$$

Acknowledgements. The author would like to thank the referee for many helpful comments.

This material is based on work supported by the U.S.-Yugoslav Joint Fund for Scientific and Technological Co-operation, in co-operation with NSF under grant JFP 544.

\section{REFERENCES} 319.

1. J. J. Benedetto, Analytic representation of generalized functions, Math. Z. 97 (1967), 303-

2. A. Byrne and E. R. Love, Complex inversion theorems for generalized Stieltjes transforms, J. Australian Math. Soc. 18 (1974), 328-358.

3. R. D. Carmichael and E. O. Milton, Abelian Theorems for the distributional Stieltjes transforms, J. Math. Anal. Appl. 72 (1979), 195-205.

4. I. M. Gelfand and G. E. Shilov, Generalized Functions, Vol. 1 (Academic Press, New York, 1964).

5. A. ERdELYI, Stieltjes transform of generalized functions, Proc. Royal Soc. Edinburgh 76A (1977), 231-249.

6. I. I. Hirschman and D. V. Widder, The convolution transform (Princeton University Press, Princeton, 1955).

7. J. Lavoine and O. P. Misra, Théorèmes abéliens pour la transformation de Stieltjes des distributions, C.R. Acad. Sci. Paris, Série A 279 (1974), 99-102.

8. J. Lavoine and O. P. MISRA, Abelian theorems for the distributional Stieltjes Transformation, Math. Proc. Camb. Philos. Soc. 86 (1979), 287-293.

9. E. R. Love and A. Byrne, Real inversion theorems for generalized Stieltjes transforms, $J$. London Math. Soc. (2) 22 (1980), 285-306.

10. O. P. MisRa, Some Abelian theorems for distributional Stieltjes transformation, J. Math. Anal. Appl. 39 (1972), 590-599.

11. J. N. Pandey, On the Stieltjes transform of generalized functions, Math. Proc. Camb. Philos. Soc. 71 (1971), 85-96.

12. R. S. Pathak, A distributional generalized Stieltjes transformation, Proc. Edinburgh Math. Soc. 20 (1976), 15-22.

13. D. B. Sumner, An inversion formula for the generalized Stieltjes transform, Bul. Amer. Math. Soc. 55 (1949), 174-183.

14. V. S. Vladimirov, Generalized Functions in Mathematical Physics (MIR Publishers, Moscow, 1979).

15. D. V. WIdder, The Laplace Transform (Princeton University Press, Princeton, N.J., 1946).

16. A. H. Zemanian, Generalized Integral Transformations (Interscience, New York, 1968).

INSTITUTE OF MATHEMATICS

21000 Novi SAD

Yugoslavia 